\title{
Spectroscopic Sensing Method of Liquid Permittivity with On-Chip Capacitor
}

\author{
Hongkie $\operatorname{Lim}^{1} \cdot$ Dong-Ho Lee ${ }^{2} \cdot$ Jusung Kim ${ }^{3, *} \cdot$ Songcheol Hong ${ }^{1}$
}

\begin{abstract}
A liquid permittivity sensing method is proposed, which is based on measuring the $S_{21}$ of an on-chip capacitor submerged in a material under test (MUT). The real part of permittivity can be estimated from $\varepsilon^{\prime}-\left|S_{21}\right|$ fitted relation in the frequency range of $100 \mathrm{MHz}-5$ $\mathrm{GHz}$, which are pre-calibrated with four kinds of known materials. The on-chip capacitor is realized with a top metal in a complementary metal oxide semiconductor (CMOS) process, which has an inter-digitized structure featuring a small size of $150 \mu \mathrm{m} \times 195 \mu \mathrm{m}$. A simple circuit model of the capacitor is used to develop $\varepsilon^{\prime}-\left|S_{21}\right|$ relation with a fitting parameter. It shows less than $6.4 \%$ root-mean-square (rms) error of $\varepsilon^{\prime}$ for propanol $\left(\mathrm{C}_{3} \mathrm{H}_{8} \mathrm{O}\right)$ at $100 \mathrm{MHz}-5 \mathrm{GHz}$.
\end{abstract}

Key Words: Calibration, Capacitive Sensor, Curve Fitting, Dielectric Spectroscopy, Inter-Digitized.

\section{INTRODUCTION}

Dielectric properties (e.g., permittivity) of chemical and biomolecules is useful for a variety of applications. In agriculture, the quality of its products is improved by measuring the permittivity of soil and leaves $[1,2]$. For example, crop quality control is usually carried out by regularly monitoring the water content of crops [1]. The dielectric characterization is also employed in biomedical sensors, such as the monitoring of blood glucose and semen activity [3-6].

In [7], a complementary metal oxide semiconductor (CMOS) based transceiver IC is presented as a dielectric spectroscopy device, but an off-chip sensing capacitor is utilized to measure the off-chip liquid material under test (MUT). This off-chip sensing capacitor is implemented on the printed circuit board (PCB) with a bulky footprint and accompanying parasitics de- grading the measurement accuracy. In [8], a PCB-integrated microwave sensor for the dielectric measurement of liquids is presented. The resonant frequency and the $\mathrm{Q}$ factor of the offchip microwave sensor are used to estimate the complex permittivity of the material to be tested. However, the work in [8] also suffers from a large area, and additional packaging processes increase the cost of the permittivity sensor. Thus, integrated sensors on silicon to detect the permittivity of chemical and biochemical are promising with a reduction in its size and cost while offering lower power consumption [9].

Capacitance can be determined if the geometry of the conductor and the dielectric properties of the insulator between the conductors are given [10]. Then, dielectric spectroscopy is accomplished by measuring the admittance (or impedance) of the sensing capacitor whose dielectric constant is dictated by the MUT and extract its dielectric constant from the material de-

Manuscript received December 23, 2020 ; Revised April 28, 2021 ; Accepted June 1, 2021. (ID No. 20201223-211J)

${ }^{1}$ Department of Electrical Engineering, Korea Advanced Institute of Science and Technology (KAIST), Daejeon, Korea.

${ }^{2}$ Department of Information and Communication Engineering, Hanbat National University, Daejeon, Korea.

${ }^{3}$ Department of Electronics Engineering, Hanbat National University, Daejeon, Korea.

"Corresponding Author: Jusung Kim (e-mail: jusungkim@hanbat.ac.kr)

This is an Open-Access article distributed under the terms of the Creative Commons Attribution Non-Commercial License (http://creativecommons.org/licenses/by-nc/4.0) which permits unrestricted non-commercial use, distribution, and reproduction in any medium, provided the original work is properly cited.

(c) Copyright The Korean Institute of Electromagnetic Engineering and Science. 
pendent capacitance.

A sensing capacitor, as a critical interface between the MUT and the spectroscopy measurement system can be implemented in either on-chip or off-chip [7-9,11]. As mentioned earlier, an off-chip sensing capacitor suffers from large parasitics and its bulky size. The interconnection between an off-chip capacitor and a spectroscopy device requires a careful attention as well.

In this work, we propose a design and am implementation of an on-chip capacitor for dielectric constant extraction featuring a small sensor size and lower parasitic, and built a low-cost. Utilizing the top metal in the CMOS back-end, inter-digitized conductors are fabricated using 28-nm CMOS technology. The dielectric properties between inter-digitized conductors are governed by the off-chip liquid MUTs by opening the passivation layer. The permittivity of the MUT is extracted within the 100 $\mathrm{MHz}_{-}-5 \mathrm{GHz}$ frequency range by measuring the forward voltage-gain $\left(S_{21}\right)$. Permittivity as a function of $\left|S_{21}\right|$ at each frequency can be extracted by polynomial curve fitting. Additionally, we propose the weighted $\left|S_{21}\right|$ equation to improve a sensor accuracy. Through a proposed curve-fitting calibration method, less than $6.4 \%$ root-mean-square (rms) error is successfully achieved when propanol $\left(\mathrm{C}_{3} \mathrm{H}_{8} \mathrm{O}\right)$ is used as a material.

This article is organized as follows. Section II discusses a simple but intuitive circuit model for the sensing capacitor. Section III presents measurement results and polynomial curve fitting method for calibration. The conclusion of this article is in Section IV.

\section{CiRCUIT MODEL OF SENSING CAPACITOR}

Fig. 1 shows the illustration of an on-chip sensing capacitor whose dielectric property is governed by the MUT. The sensing capacitor is in an inter-digitized fashion implemented with the top metal of the CMOS back-end process (LB in the 28-nm Samsung CMOS process). The passivation layer has to be removed in order to contact the top metal directly to MUT.

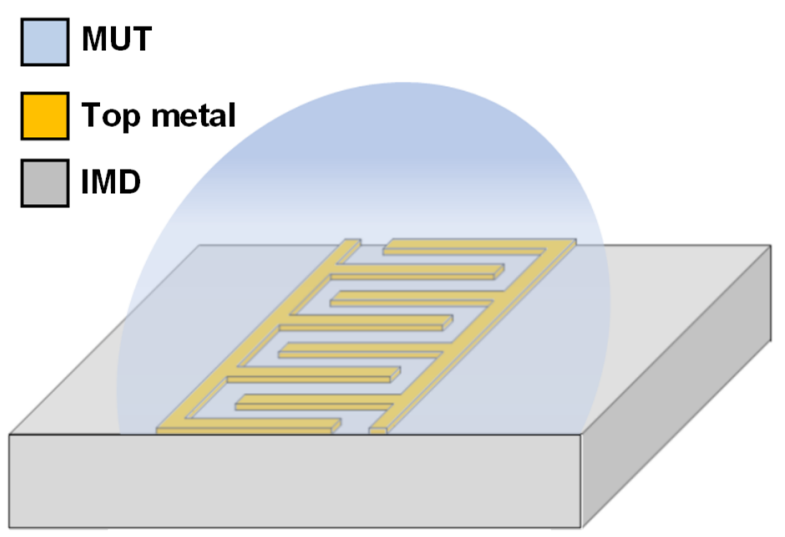

Fig. 1. Illustration of an inter-digitized top-metal as an on-chip sensing capacitor covered by a material under test (MUT).
A side section view of the on-chip sensing capacitor with a simple equivalent circuit model is depicted in Fig. 2. Capacitance and conductance denoted as $\mathrm{C}_{\text {sensing }}$ and $\mathrm{G}_{\text {sensing }}$ are due to the E-fields passing through the MUT by two inter-digitized top metal nodes, while the E-fields passing through the intermetal-dielectric (IMD) create undesired parasitic capacitance and conductance modeled as $\mathrm{C}_{\mathrm{par}}$ and $\mathrm{G}_{\mathrm{par}}$, which are not related to the characteristics of the MUT. Additionally, capacitance due to the substrate, $\mathrm{C}_{\mathrm{sub}}$, is part of the network, which degrades the accuracy of the permittivity detection.

In the case of $\mathrm{C}_{\text {sensing }}$ and $\mathrm{G}_{\text {sensing }}$, their values are determined according to the permittivity of the MUT $\left(\varepsilon_{M U T}=\varepsilon_{M U T}{ }^{\prime}-j\right.$. $\left.\varepsilon_{M U T^{\prime}}\right)$. In contrast, $\mathrm{C}_{\mathrm{par}}$ and $\mathrm{G}_{\mathrm{par}}$ are fixed values governed by permittivity of the $\operatorname{IMD}\left(\varepsilon_{I M D}=\varepsilon_{I M D^{\prime}}-j \cdot \varepsilon_{I M D^{\prime}}\right)$.

Excluding the effect of $\mathrm{C}_{\text {sub }}$, the real-part of admittance between two inter-digitized electrodes $\left(Y_{S}\right)$ comes from $\mathrm{G}_{\text {sensing }}$ and $\mathrm{G}_{\mathrm{par}}$, while the imaginary part of $Y_{S}$ is composed of $\mathrm{C}_{\text {sensing }}$ and $\mathrm{C}_{\text {par }} \cdot \mathrm{G}_{\text {sensing }}$ and $\mathrm{G}_{\mathrm{par}}$ are circuit components due to the imaginary permittivity of MUT $\varepsilon_{M U T}$ " and IMD $\varepsilon_{I M D}$ ", respectively, which corresponds to an energy loss. $\mathrm{C}_{\text {sensing }}$ and $\mathrm{C}_{\mathrm{par}}$ come from the real permittivity of MUT $\varepsilon_{M U T}{ }^{\prime}$ and IMD $\varepsilon_{I M D}$ ', respectively, representing energy storage [12]. Combined admittance between two electrodes can be expressed as follows.

$$
\begin{aligned}
Y_{S} & =j \omega \cdot C_{0} \cdot\left(\varepsilon_{M U T}-j \cdot \varepsilon_{M U T}{ }^{\prime}\right) \\
& +j \omega \cdot C_{1} \cdot\left(\varepsilon_{I M D^{\prime}}-j \cdot \varepsilon_{I M D^{\prime}}\right) \\
& =j \omega \cdot\left(C_{0} \cdot \varepsilon_{M U T^{\prime}}+C_{1} \cdot \varepsilon_{I M D^{\prime}}\right) \\
& +\omega \cdot\left(C_{0} \cdot \varepsilon_{M U T^{\prime \prime}}+C_{1} \cdot \varepsilon_{I M D^{\prime \prime}}\right) \\
& =j \omega \cdot f\left(\varepsilon_{M U T^{\prime}}\right)+\omega \cdot g\left(\varepsilon_{M U T}{ }^{\prime \prime}\right)
\end{aligned}
$$

where $C_{0}\left(C_{1}\right)$ are coefficients of effective capacitance due to MUT and IMD and $f(\cdot)$ and $g(\cdot)$ denote nonlinear mapping functions from the permittivity of MUT to admittance as a result.

To improve the sensitivity in measuring the permittivity of MUT, parasitic capacitances, $\mathrm{C}_{\mathrm{par}}$ and $\mathrm{C}_{\text {sub }}$ in parallel with the

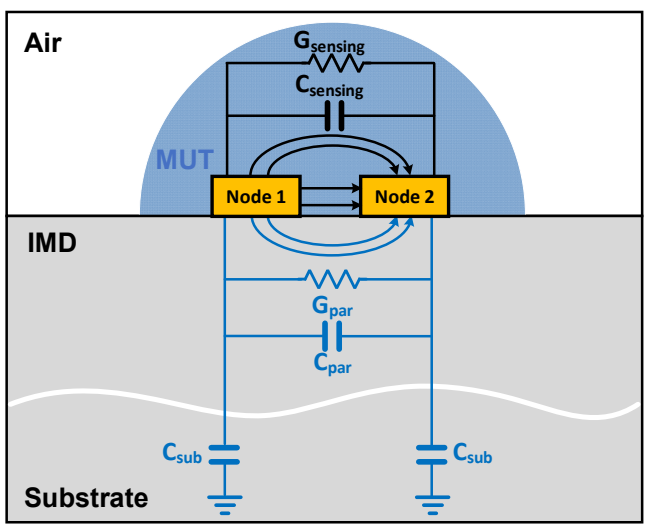

Fig. 2. Side section view of a sensing capacitor with a simple equivalent circuit model. 
desired capacitance, $\mathrm{C}_{\text {sensing }}$, have to be minimized. $\mathrm{C}_{\text {sub }}$ can be made small by narrowing the spacing of the two nodes of the sensing capacitor. At the same time, narrow spacing between two electrodes reduces the self-resonant frequency (SRF) as a result of an increase in $\mathrm{C}_{\text {sensing }}$ and mutual inductances. The inductances caused by the geometry of the on-chip sensing capacitor have a direct trade-offs with the size of the sensing capacitor such as a spacing and the width of the top metal. Thus, in this work, the target frequency band and area of the chip are budgeted in advance, and the optimal sensing capacitor dimension is determined in consideration of the above mentioned trade-offs.

The top metal is composed of aluminum, and an oxide layer is created when the top metal is exposed to the MUT without any passivation (protection). The thick oxide film attenuates the electric field and reduces the sensitivity of the sensing capacitor. In the fabricated chip, the oxide film thickness ranges from 1 to $5 \mathrm{~nm}$ [13]. The electromagnetic simulation was performed and the sensitivity was not degraded even with $10 \mathrm{~nm}$ oxide film thickness. This thin oxide film, on the other hand, is beneficial by protecting the sensing capacitor from the damage and chemical reaction due to its high corrosion resistance and wear resistance.

Fig. 3 shows the schematic model of the sensing area with a two-port vector network analyzer (VNA). The forward voltage gain $\left(S_{21}\right)$ of this network can be calculated by finding the ratio between the injected voltage source $\left(V_{1}\right)$ and the load voltage $\left(V_{2}\right)$ while, the network is terminated with the port load impedance $\left(R_{\text {port }}\right)$.

$$
\begin{aligned}
S_{21}(j \omega)=\frac{V_{2}}{V_{1}}= & \frac{Y_{S}}{Y_{S}+\frac{1}{R_{p o r t}}+j \omega \cdot C_{s u b}} \\
& =\frac{\omega \cdot g\left(\varepsilon_{M U T^{\prime \prime}}\right)+j \omega \cdot f\left(\varepsilon_{M U T^{\prime}}\right)}{\omega \cdot g\left(\varepsilon_{M U T^{\prime \prime}}\right)+\frac{1}{R_{\text {port }}}+j \omega \cdot\left(f\left(\varepsilon_{M U T^{\prime}}\right)+C_{\text {sub }}\right)}
\end{aligned}
$$

In both the numerator and the denominator, the resistance $\left(\omega \cdot g\left(\varepsilon_{M U T^{\prime \prime}}\right)\right)$ due to the imaginary part of permittivity is significantly smaller than those of the other components. For in-

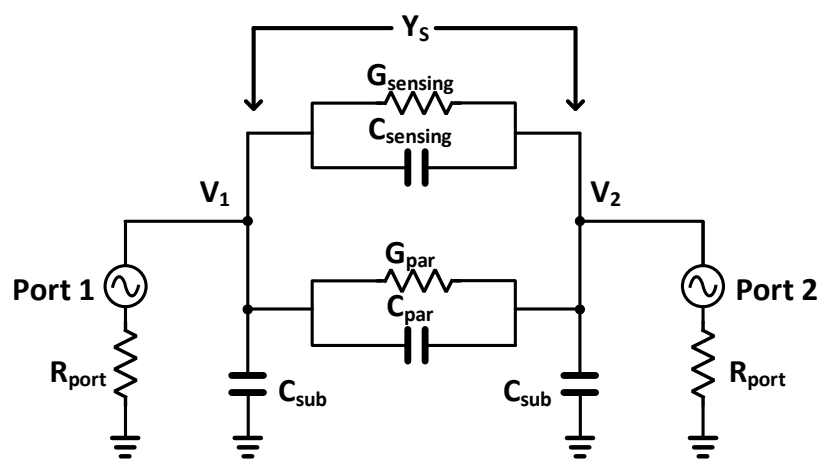

Fig. 3. Schematic model of sensing capacitor and two-port network vector network analyzer (VNA). stance, long transmission lines, connected in series with a sensing capacitor, makes $Y_{S}$ more capacitive. Thus, we can simplify Eq. (2) into the following formula:

$$
S_{21}(j \omega) \cong \frac{j \omega \cdot R_{\text {port }} \cdot f\left(\varepsilon_{M U T^{\prime}}\right)}{1+j \omega \cdot R_{\text {port }} \cdot\left(f\left(\varepsilon_{M U T^{\prime}}\right)+C_{\text {sub }}\right)} .
$$

The dominant pole is calculated to be $\omega_{p}=\frac{1}{R_{\text {port }} \cdot\left(f\left(\varepsilon_{\text {Mut }}\right)+C_{\text {sub }}\right)}$. Electromagnetic simulation with Advanced Design System (ADS) shows that $\omega_{p}$ is higher than $6 \mathrm{GHz}$. Accordingly, in the expected measurement range between 0.1 and $5 \mathrm{GHz}$, the magnitude of $S_{21}$ can be written as $\left|S_{21}(j \omega)\right| \cong \omega \cdot R_{\text {port }}$. $f\left(\varepsilon_{M U T}{ }^{\prime}\right)$. This equation shows that magnitude of $S_{21}$ indicates the real part of the permittivity of $\operatorname{MUT}\left(\varepsilon_{M U T}\right)$, while the nonlinear mapping function, $f(\cdot)$, needs to be decoded in a certain manner, as discussed in Section III.

\section{MEASUREMENT RESULTS AND CURVE FitTiNG \\ CALIBRATION}

For the measurement of scattering parameters of the fabricated sensor, $\mathrm{PCB}$ consisting of the mounted chip, interconnect transmission lines, and input/output ports are assembled as shown in Fig. 4. During the chip packaging process, the insulating epoxy was covered except for the fabricated capacitor area. With a plastic tube on top of the chip, the MUTs were injected through the micropipette (Eppendorf Research plus 3120000038). Fig. 5 shows the real part of the permittivity $\left(\varepsilon^{\prime}\right)$ for methanol, ethanol, propanol, butanol and air over the frequency range of $0.1-5$

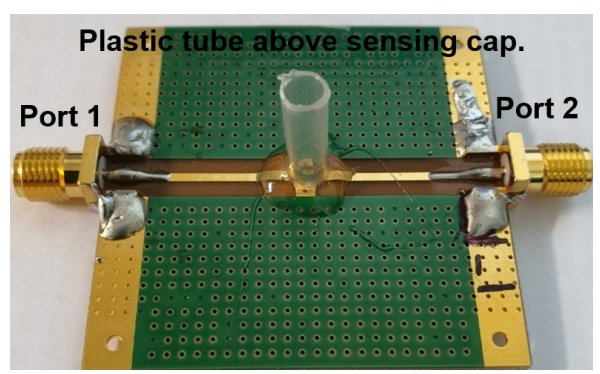

Fig. 4. Photograph of measurement set up.

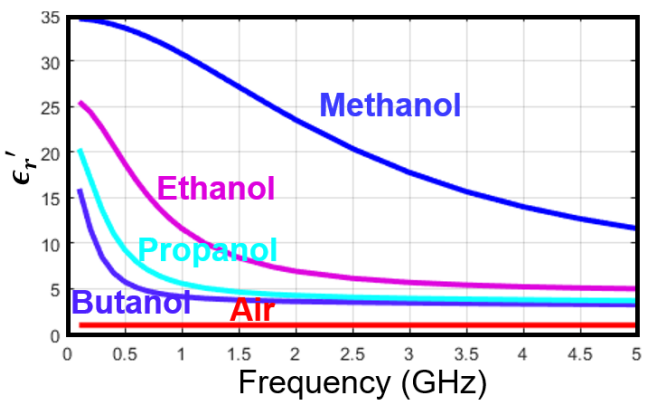

Fig. 5. Real-part of permittivity $\varepsilon^{\prime}$ of MUTs over the frequency range of $0.1-5 \mathrm{GHz}$ [14]. 
$\mathrm{GHz}$ [14]. The measured forward voltage gain $\left(S_{21}\right)$ at room temperature is given in Fig. 6, which shows that the magnitude of $S_{21}$ is proportional to $\varepsilon^{\prime}$ over the measured frequency range.

To extract $\varepsilon^{\prime}$ of the MUT from the measured $S_{21}$ data, the curve-fitting calibration is performed first. Methanol, ethanol, butanol, and air are selected as reference materials, where the information on their real permittivity is provided in [14]. The real permittivity is then curve-fitted at each measured frequency by third order polynomial function with the measured $\left|S_{21}\right|$.

The detailed polynomial curve fitting procedure from the measured $\left|S_{21}\right|$ is elaborated below:

1. For each frequency, $f_{1}$, to be curve fitted, reference materials are injected into the sensing capacitor and its $\left|S_{21}\right|$ is measured through a VNA.

2. The $\varepsilon^{\prime}$ of the reference materials are least-square fitted to third order polynomial function with respect to the measured $\left|S_{21}\right|$.

$$
\varepsilon^{\prime}=a_{3}\left|S_{21}\right|^{3}+a_{2}\left|S_{21}\right|^{2}+a_{1}\left|S_{21}\right|+a_{0}
$$

3. The fitting parameters $a_{0}, a_{1}, a_{2}$, and $a_{3}$ can be found and saved by using the least-square fitting method to fit the polynomial regression function. This procedure is repeated over the entire measurement frequency.

Once the curve-fitting procedure is finished, the unknown permittivity of MUT can be calculated by utilizing its measured $\left|S_{21}\right|$ along with the calculated fitting parameters.

Given the above procedure, the permittivity of any unknown MUT can be extracted. To further enhance the measurement accuracy, we propose reshaping (weighting) the parameter, given the measured $S_{21}$. Instead of utilizing raw $\left|S_{21}\right|$, we give the weights $\beta$ on the imaginary part of $S_{21}$ and $(1-\beta)$ on the real part of $S_{21}$. The weighting function can emphasize/deemphasize the relevant terms to minimize the extraction error. The revised magnitude of $S_{21}$ is given by

$$
\begin{aligned}
& \left|S_{21}\right|^{*}=\sqrt{(1-\beta) \cdot \operatorname{Real}\left(S_{21}\right)^{2}+\beta \cdot \operatorname{Imag}\left(S_{21}\right)^{2}}, \\
& 0 \leq \beta \leq 1
\end{aligned}
$$

Fig. 7 shows the curve-fitted polynomial function and the real permittivity of the reference materials at $100 \mathrm{MHz}$ and $5 \mathrm{GHz}$ frequency with the frequency-dependent parameter $\beta$. From the extracted curve-fitted polynomial function, the permittivity of propanol is found to have a good correlation with reference value as shown in Fig. 8. By replacing $\left|S_{21}\right|$ with the proposed equation, the rms permittivity error of measurement is lowered from $9.1 \%$ to $6.4 \%$ over the frequency range of $0.1-5 \mathrm{GHz}$.

The proposed spectroscopy sensor is compared to the reported research works as depicted in Table 1. Our work is realized in a compact footprint $\left(0.03 \mathrm{~mm}^{2}\right)$ on chip, while the operating frequency range is the second largest.

More reference materials for calibration can be added to minimize the permittivity error (higher polynomial function). The off-chip and on-chip transmission line in series with the sensing capacitor complicate the mapping function and degrade the accuracy accordingly. Their length then needs to be minimized to further enhance the measurement accuracy.

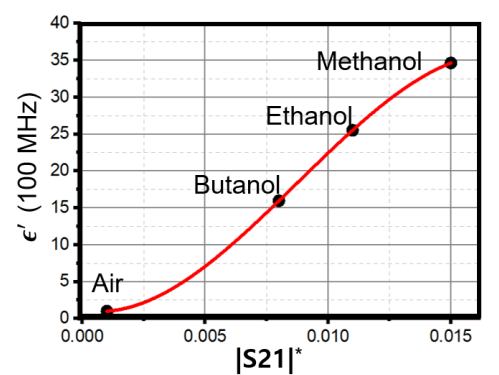

(a)

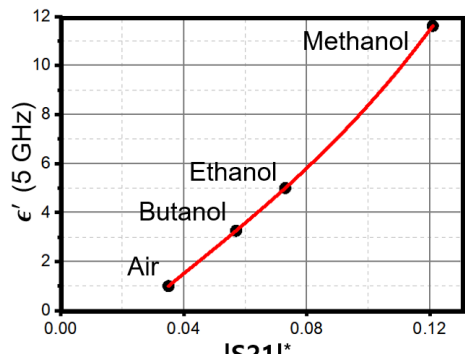

(b)

Fig. 7. $\varepsilon^{\prime}-\left|S_{21}\right|^{*}$ curve-fitted with known $\varepsilon^{\prime}$ of four reference materials at (a) $100 \mathrm{MHz}$ and (b) $5 \mathrm{GHz}$.

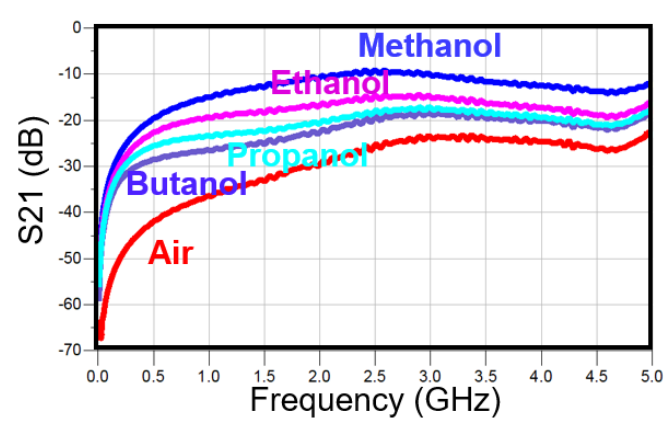

Fig. 6. Measured $\left|S_{21}\right|$ of sensing capacitor over the frequency range of $0.1-5 \mathrm{GHz}$.

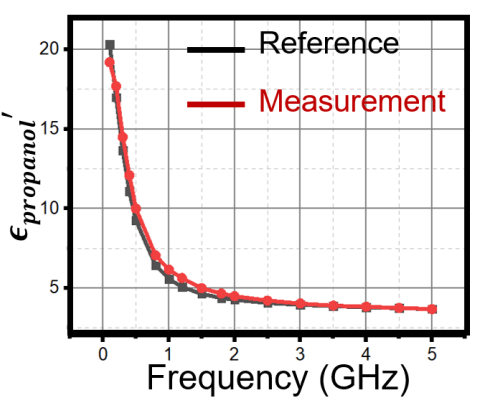

Fig. 8. Measured $\varepsilon^{\prime}$ of propanol with the proposed fitting method and reference value. 
Table 1. Comparison of the proposed spectroscopy sensor with previous works

\begin{tabular}{lccc}
\hline \multicolumn{1}{c}{ Study } & $\begin{array}{c}\text { Frequency } \\
(\mathrm{GHz})\end{array}$ & Architecture & $\begin{array}{c}\text { Size } \\
\left(\mathrm{mm}^{2}\right)\end{array}$ \\
\hline Bakhshiani et al. [7] & $0.009-2.4$ & $\begin{array}{c}\text { Off-chip center tapped } \\
\text { microstrip line }\end{array}$ & N/A \\
Chuma et al. [8] & 2.4 & Off-chip split ring resonator & N/A \\
Helmy et al. [9] & $7-9$ & On-chip inter-digitized & 0.06 \\
capacitor & 0.03 \\
Chien et al. [11] & $1-50$ & On-chip transmission line & 0.03 \\
This work & $0.1-5$ & On-chip inter-digitized & 0.03 \\
& \multicolumn{3}{c}{ capacitor } \\
\hline
\end{tabular}

\section{CONCLUSION}

An on-chip capacitive sensor for dielectric spectroscopy is implemented in this work. Based on the circuit model of the on-chip sensing capacitor, the permittivity extraction method is proposed and the measurement result verifies the successful detection of the real part of the permittivity in the frequency range of $0.1-5 \mathrm{GHz}$. The inter-digitized sensing capacitor can be optimized in its sensitivity and SRF in consideration of the fabrication trade-offs. To improve the permittivity detection accuracy, the measured forward voltage-gain is curve-fitted by the polynomial regression function. The revised forward voltage-gain parameter is suggested for the detection and the measured permittivity of the propanol shows $6.4 \%$ rms error compared with the theoretical value.

This work was supported by the National Research Foundation of Korea (NRF) grant funded by the Korea Government (MSIP) (No. NRF-2019R1F1A1048784) and Samsung Electronics Co., Ltd.

\section{REFERENCES}

[1] K. B. Kim, J. H. Kim, S. S. Lee, and S. H. Noh, "Measurement of grain moisture content using microwave attenuation at $10.5 \mathrm{GHz}$ and moisture density," IEEE Transactions on Instrumentation and Measurement, vol. 51, no. 1, pp. 72-77, 2002.

[2] H. M. Al-Mattarneh, D. K. Ghodgaonkar, and W. M. W. Majid, "Microwave nondestructive testing for classification of Malaysian timber using free-space techniques," in Proceedings of the 6th International Symposium on Signal Processing and its Applications (Cat. No. 01EX467), Kuala Lumpur, Malaysia, 2001, pp. 450-453.
[3] A. Lonappan, G. N. Bindu, and V. Thomas, and K. Mathew, "Analysis of human semen using microwaves," Progress In Electromagnetics Research, vol. 57, pp. 277-284, 2006.

[4] A. Lonappan, V. Thomas, G. Bindu, C. Rajasekaran, and K. T. Mathew, "Nondestructive measurement of human blood at microwave frequencies," Journal of Electromagnetic Waves and Applications, vol. 21, no. 8, pp. 1131-1139, 2007.

[5] H. F. Cook, "Dielectric behaviour of human blood at microwave frequencies," Nature, vol. 168, no. 4267, pp. 247248, 1951.

[6] Y. Hayashi, L. Livshits, A. Caduff, and Y. Feldman, "Dielectric spectroscopy study of specific glucose influence on human erythrocyte membranes," Journal of Physics D: Applied Physics, vol. 36, no. 4, pp. 369-374, 2003.

[7] M. Bakhshiani, M. A. Suster, and P. Mohseni, "A 9 MHz$2.4 \mathrm{GHz}$ fully integrated transceiver IC for a microfluidicCMOS platform dedicated to miniaturized dielectric spectroscopy," IEEE Transactions on Biomedical Circuits and Systems, vol. 9, no. 6, pp. 849-861, 2015.

[8] E. L. Chuma, Y. Iano, G. Fontgalland, L. L. B. Roger, and H. Loschi, "PCB-integrated non-destructive microwave sensor for liquid dielectric spectroscopy based on planar metamaterial resonator," Sensors and Actuators A: Physical, vol. 312, article no. 112112, 2020. https://doi.org/10.1016/ j.sna.2020.112112

[9] A. A. Helmy, H. J. Jeon, Y. C. Lo, A. J. Larsson, R. Kulkarni, J. Kim, J. Silva-Martinez, and K. Entesari, "A selfsustained CMOS microwave chemical sensor using a frequency synthesizer," IEEE Journal of Solid-State Circuits, vol. 47, no. 10, pp. 2467-2483, 2012.

[10] D. M. Pozar, Microwave Engineering. Hoboken, NJ: John Wiley \& Sons, 2004.

[11] J. C. Chien, M. Anwar, E. C. Yeh, L. P. Lee, and A. M. Niknejad, "A 1-50 GHz dielectric spectroscopy biosensor with integrated receiver front-end in 65nm CMOS," in Proceedings of 2013 IEEE MTT-S International Microwave Symposium Digest (MTT), Seattle, WA, 2013, pp. 1-4.

[12] M. M. Bajestan, A. A. Helmy, H. Hedayati, and K. Entesari, "A $0.62-10 \mathrm{GHz}$ complex dielectric spectroscopy system in CMOS," IEEE Transactions on Microwave Theory and Techniques, vol. 62, no. 12, pp. 3522-3537, 2014.

[13] M. S. Hunter and P. Fowle, "Natural and thermally formed oxide films on aluminum," Journal of the Electrochemical Society, vol. 103, no. 9, pp. 482-485, 1956.

[14] A. P. Gregory and R. N. Clarke, "Tables of the complex permittivity of dielectric reference liquids at frequencies up to $5 \mathrm{GHz}$," National Physical Laboratory, Middlesex, UK, NPL Report. MAT23, 2012. 
Hongkie Lim

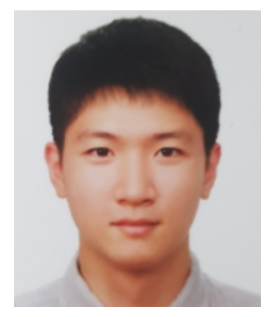

received B.S. degrees in School of Electrical and Electronics Engineering, Chung-Ang University, Seoul, South Korea in 2019 and M.S. degree in the Korea Advanced Institute of Science and Technology, Daejeon, South Korea, in 2021. Since 2021, he is currently with Samsung Electronics, Co., Ltd., Hwaseong, South Korea as an engineer. His research interests include radio frequency $(\mathrm{RF})$ circuit and dielectric spectroscopy.
Jusung Kim

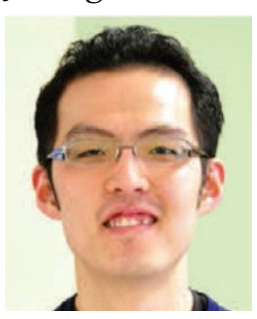

received a B.S. degree (with highest honors) from Yonsei University, Seoul, South Korea, in 2006, and a Ph.D. degree from Texas A\&M University, College Station, TX, USA, in 2011, both in electrical engineering. In 2008, he was employed as an Analog Integrated Circuit Design Engineer at Texas Instruments Inc., Dallas, TX, USA, where he designed an RF front end for a multi standard analog and digital TV silicon tuners. From 2011 to 2015, he was with the Qualcomm Technologies Inc., San Diego, CA, USA, where he designed radio frequency integrated circuits (RFIC) products for $3 \mathrm{G}$ and $4 \mathrm{G}$ cellular systems. $\mathrm{He}$ is currently an associate professor with the Department of Electronics and Control Engineering, Hanbat National University, Daejeon, South Korea. His current research focuses on the design and fabrication of lowpower integrated circuits for communication and biomedical applications. Dr. Kim is an Analog Signal Processing Technical Committee member of the IEEE Circuits and Systems Society. He was an associate editor for the IEEE Transaction on Circuits and Systems-II: Express Briefs from 2014 to 2015 .

\section{Songcheol Hong}

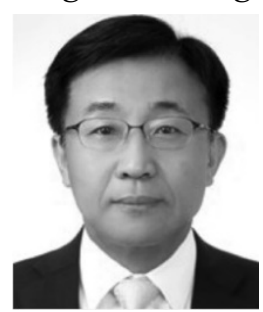

received the B.S. and M.S. degrees in electronics engineering from Seoul National University, Seoul, South Korea, in 1982 and 1984, respectively, and the Ph.D. degree in electrical engineering and computer science from the University of Michigan, Ann Arbor, MI, USA, in 1989. In 1997, he joined the EECS Department, Stanford University, Stanford, CA, USA, as a visiting professor. He worked with Samsung Microwave Semiconductor, Milpitas, CA, USA. He served as the Dean of Research Affairs and the Director of KI-IT Convergence with the Korea Advanced Institute of Science and Technology (KAIST), Daejeon, South Korea. He is currently a professor with the School of Electrical Engineering, KAIST, where he is also a KT-Chaired Professor. He has authored or coauthored more than 300 technical papers and 150 patents. His current research interests include RFICs and RF CMOS PAs, especially in millimeter-wave ICs for $5 \mathrm{G}$ communications and radars. Dr. Hong is currently a member of NAEK, KIEES, and KITE. He served as a Board member of Techno-park of Daejeon Metropolitan city. He served as the General Chair for RFIT 2017 supported by the IEEE and the TPC Chair for APMC 2013 and GSMM 2014. 